\title{
Density Normalization of Susceptibility for Compressible Artificial Material Measurements
}

\author{
Logan A. Loeb ${ }^{*}$ and Keith W. Whites \\ Laboratory for Applied Electromagnetics and Communications \\ Department of Electrical and Computer Engineering \\ South Dakota School of Mines and Technology \\ Rapid City, South Dakota 57701
}

\section{INTRODUCTION}

Knowledge of the electromagnetic characteristics and properties of materials at high frequencies is critical to numerous research areas and industrial applications. In many methods of material characterization, preparing the specimen under test requires as much, if not more, care than the measurement itself. The method described here has used a coaxial clam-shell fixture to measure the complex permittivity of composite dielectric materials. This fixture can potentially compress a specimen, which has been seen to increase the extracted permittivity above that of an uncompressed specimen. We propose that this change can be compensated for by normalizing the measured electric susceptibility to the material's density while the specimen is compressed in the test fixture.

\section{MATERIAL MEASUREMENTS}

The test fixture used in our material characterizations was the Damaskos, Inc. Measurement Fixture M07T, which is illustrated in Fig. 1. It is a $7 \mathrm{~mm}$ airline fixture consisting of a center conductor and an outer conductor that splits in half lengthwise for easy insertion and removal of specimens.

The permittivities of the specimens were obtained with the MU-EPSLN ${ }^{\text {TM }}$ Coaxial Line and Waveguide Material Measurements and Processing Software from Damaskos, Inc. and an Agilent E8364B PNA Network Analyzer. Permittivity values were extracted via the "Eps from Transmission" reduction algorithm included in the software, which is equivalent to the NIST Transmission/Reflection extraction method with $\beta$ equal to zero [1], [2]. Since transmission line extraction methods lack sensitivity to low-loss materials like those investigated here, the following discussion focuses only on the real part of their measured permittivities.

Before measuring a specimen, it is usually important to precisely machine it for a tight fit in the text fixture. Any air gaps between the specimen and conductors will adversely affect the measurement results and should be avoided. However, the materials studied here are composites of low permittivity Sylgard $\AA 184$ silicone encapsulant [3] and high permittivity strontium titanate $\left(\mathrm{SrTiO}_{3}\right)$ powder. This material can be cast directly in the test fixture to minimize air gaps during measurement and eliminate the need for precision machining.

Direct casting of this material in a mold can remove the difficulties associated with machining, but creating quality specimens can remain challenging. One obstacle is the inclusion of air bubbles in the material during the mixing process. Generally, entrained air can be removed by placing the material in a vacuum for several minutes. However, once the volume loading fraction reaches fifty percent, this approach loses its effectiveness. At this high loading, the material has a very thick consistency, and air bubbles become impossible to remove by vacuum alone. Also, injecting such a viscous material into a mold presents its own difficulties. For these reasons, data is provided to a maximum loading fraction of forty percent by volume of $\mathrm{SrTiO}_{3}$.

Because these composites have an elastomeric base, they are flexible and compressible. These properties can create issues during measurements if specimens were cast in a mold with larger (or smaller) inner dimensions than the test fixture. Such a specimen might even be very close in size to the test fixture's dimensions, but could still be slightly compressed enough during measurement to affect the extracted permittivity values.

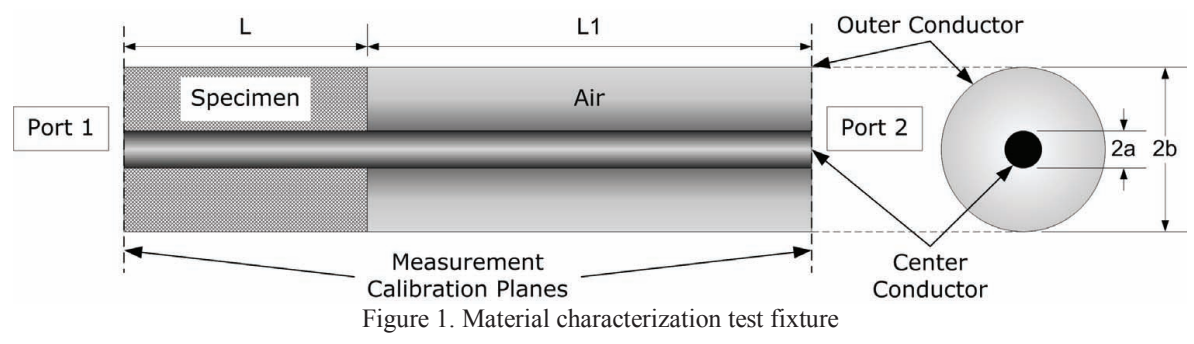

This work was supported by the National Science Foundation through an Integrative, Hybrid \& Complex Systems program grant (ECCS0824034). 


\section{DENSITY-NORMALIZED ELECTRIC SUSCEPTIBILITY}

A proposed solution to account for differences in measured and actual permittivities of specimens due to compression during measurement involves normalizing the electrical susceptibility of the extracted values to the density of the specimen. If a specimen is compressed in the test fixture, it will have a greater density than when it is out of the test fixture. As a result the measured permittivity values will be higher than the actual values. This outcome arises from the relation between electric susceptibility and polarization density given by

$$
\mathbf{P}=\varepsilon_{0} \chi_{e} \mathbf{E},
$$

where $\mathbf{P}$ is the polarization density of the material, $\chi_{e}$ is its complex electric susceptibility, $\varepsilon_{0}$ is the electric permittivity of free space, and $\mathbf{E}$ is the applied electric field [4]. Compression of a specimen increases its density of electric dipole moments, and as a result of (1) both the polarization density and electric susceptibility increase. Though not given due to our extraction method, (1) predicts that dielectric losses also increase with compression.

Having established a relationship between density and electric susceptibility, the next step is to apply it in a meaningful manner. Experimental results have shown that normalizing the measured susceptibilities of specimens in their compressed and uncompressed states to their densities in these states can noticeably diminish the effects of compression on extracted permittivity.

Density normalization begins with the fabrication of the test specimen. Here, two samples of specimens with different volume loadings of $\mathrm{SrTiO}_{3}$ were created, one cast in the test fixture and another cast in a separate mold. Since this mold was unintentionally made with a slightly larger outer diameter than the test fixture $(7.00-\mathrm{mm}$ instead of 6.9 $\mathrm{mm}$ ), specimens cast in it are compressed during permittivity measurements. Several specimens of varying lengths were created for each volume loading and molding method, and their permittivities were measured. Next, their uncompressed densities, $\rho_{u}$, were measured with a Matsuhaku GP-120S densimeter [5], which precisely measures density by the Archimedes principle. The compressed densities cannot be directly measured but can be easily estimated from

$$
\rho_{c}=\frac{m_{s}}{V_{c}},
$$

where $\rho_{c}$ is the compressed density, $m_{s}$ is the mass of the specimen, and $V_{c}$ is the volume of the specimen while in the test fixture. This volume can be found by multiplying the cross-sectional area of the specimen in the test fixture, $\pi\left(b^{2}\right.$ $a^{2}$ ), by the length, $L$, of the specimen placed inside. Note that specimen length might change between its compressed and uncompressed states.

With the above information, the density normalized susceptibilities of the specimens can be found. As the specimens cast in the test fixture were assumed to have the same densities inside and outside the test fixture $\left(\rho_{c}=\rho_{u}\right)$, their susceptibilities, $\chi_{\text {eactual }}$, are normalized to the density of uncompressed material using the following formula,

$$
\chi_{e, n o r m}=\frac{\chi_{e, a c t u a l}}{\rho_{u}} .
$$

The susceptibilities of the specimens cast in the mold, $\chi_{\text {e,compressed, }}$ can be normalized with the following two equations,

$$
\chi_{e, \text { norm }}^{c}=\frac{\chi_{e, \text { compressed }}}{\rho_{c}},
$$

and

$$
\chi_{e, \text { norm }}^{u}=\frac{\chi_{e, \text { compressed }}}{\rho_{u}},
$$

where $\chi_{e, n o r m}^{c}$ and $\chi_{e, n o r m}^{u}$ are the susceptibilities normalized to compressed and uncompressed density, respectively. Normalizing the susceptibilities removes the effect of density on the measured data.

The goal of normalizing susceptibility to density is to find the actual permittivity of a specimen that has been compressed during its measurement. For the specimen that was cast in the test fixture, there was no compression during the measurement; therefore, its measured permittivity is the actual permittivity of the material. A compressed specimen's measured permittivity is not equal to the actual permittivity of the material, but the correct value can be found from the susceptibility normalized to compressed density given in (4). Since the effects of compression have been removed by normalizing to the compressed density, the relative permittivity of the uncompressed material can be found by multiplying $\chi_{e, n o r m}^{c}$ by the uncompressed density, $\rho_{u}$, and adding one.

\section{RESULTS}

The following results show the density normalized susceptibilities of specimens at volume loading fractions ranging from 0 to $40 \%$. The figures have been truncated at frequencies corresponding to the material cutoff frequencies of higher order modes calculated by

$$
f_{c}=\frac{c k_{c}}{2 \pi \sqrt{\varepsilon_{r}}},
$$

where $k_{c}=2 /(a+b)$, as given in [4]. The susceptibilities of the specimens cast in the test fixture were normalized to their uncompressed density, which is equal to its density while in the test fixture. The dotted and dashed curves in the figures show the susceptibilities of the specimens cast in the mold normalized to their compressed and uncompressed densities, respectively.

The density-normalized susceptibilities of neat Sylgard $\mathbb{R}$ 184 specimens are shown in Fig. 2. Here, there is very little disparity between the three curves, which is an expected result, since the material has a low permittivity and does not contain any high permittivity particles. Thus, compression 
will only slightly increase the material's polarization density and susceptibility.

From the dashed curves in Fig. 4 - Fig. 7, it is plain to see that normalizing to a material's uncompressed density yields incorrect values that are significantly higher than the actual values given by the solid curves. The dotted curves, which represent normalization to a material's compressed density, more closely agree with the actual values. This result occurs because the dashed curve does not account for compression of materials during measurement. When compressed, the polarizable particles are forced closer together as in Fig. 3 and raise the effective susceptibility. The method of normalizing to compressed density compensates for this increase.

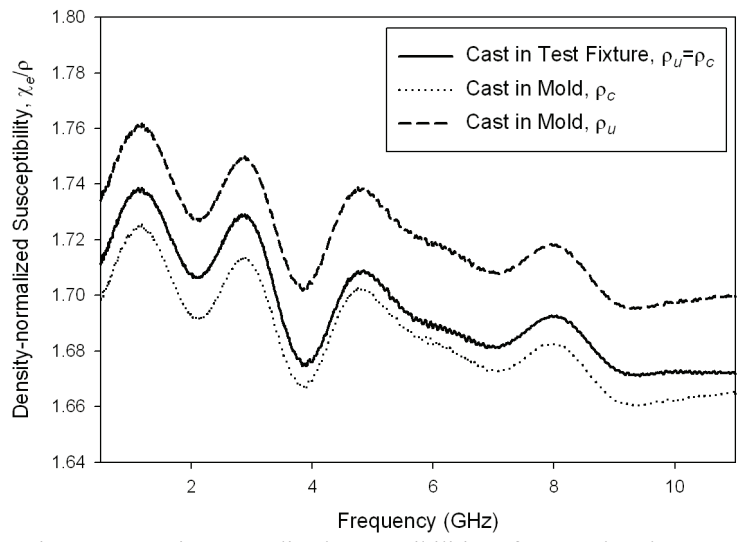

Figure 2. Density-normalized susceptibilities of neat Sylgard ${ }^{\circledR} 184$

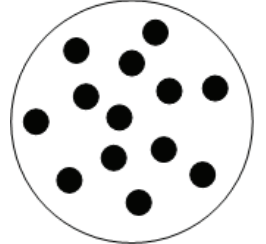

Uncompressed

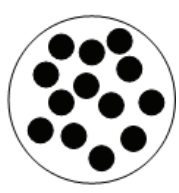

Compressed

Figure 3. An exaggerated illustration showing the effects of compression on composite materials.

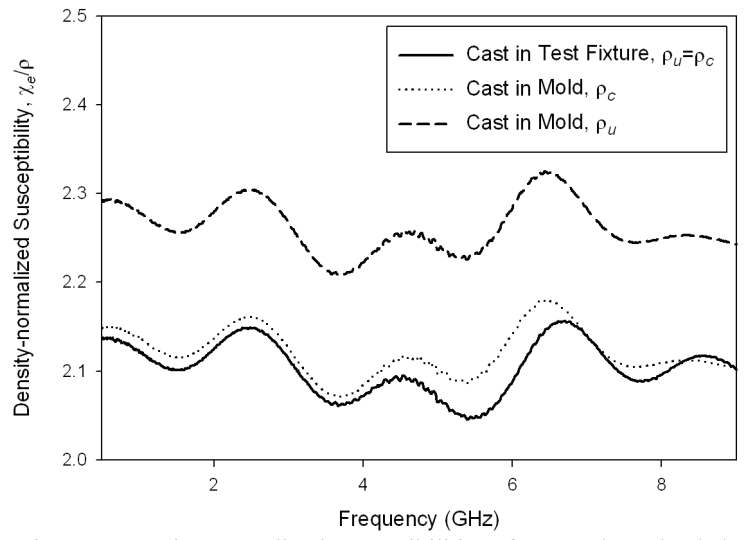

Figure 4. Density-normalized susceptibilities of $10 \%$ volume loaded material

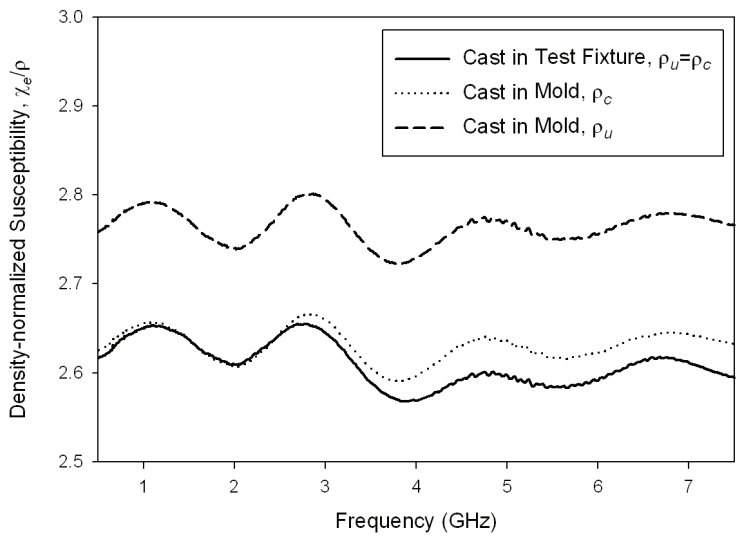

Figure 5. Density-normalized susceptibilities of $20 \%$ volume loaded material.

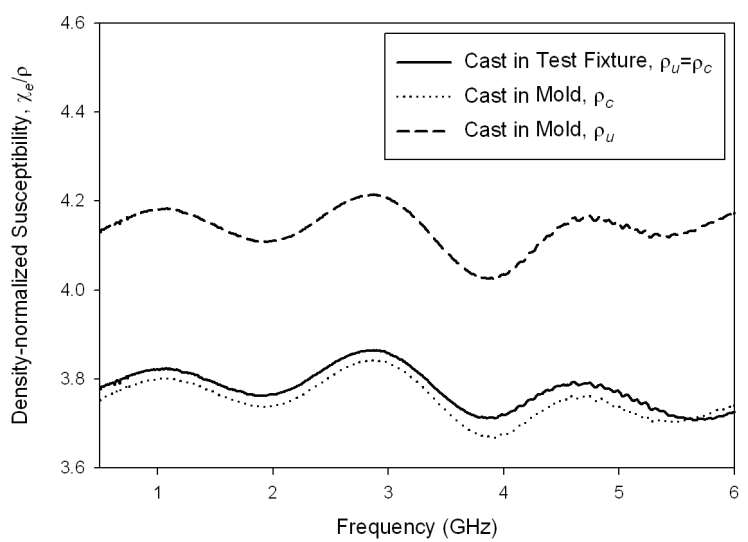

Figure 6. Density-normalized susceptibilities of $30 \%$ volume loaded material.

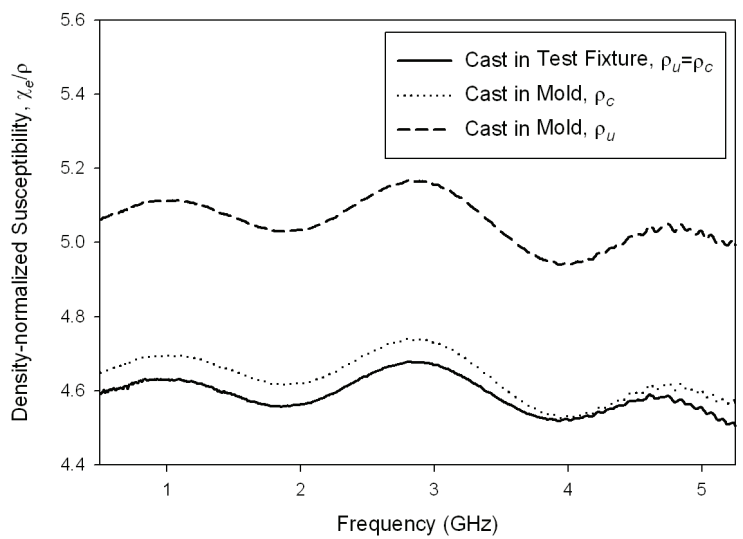

Figure 7. Density-normalized susceptibilities of $40 \%$ volume loaded material

Compensating for compression during measurement has a visible impact on the resulting permittivities of the specimens. Fig. 8 - Fig. 12 show three different permittivities for the various loading fractions. Measured permittivities of the uncompressed specimens cast in the test fixture are indicated in the figures by solid lines. These values are the actual permittivities of the materials. The 
dotted lines show the approximate permittivities after density compensation of the specimens cast in the mold. These specimens were compressed during the measurement, and their measured (uncompensated) permittivities are given by the dashed lines. Density compensation clearly yields much more accurate permittivities than direct measurement of compressed specimens.

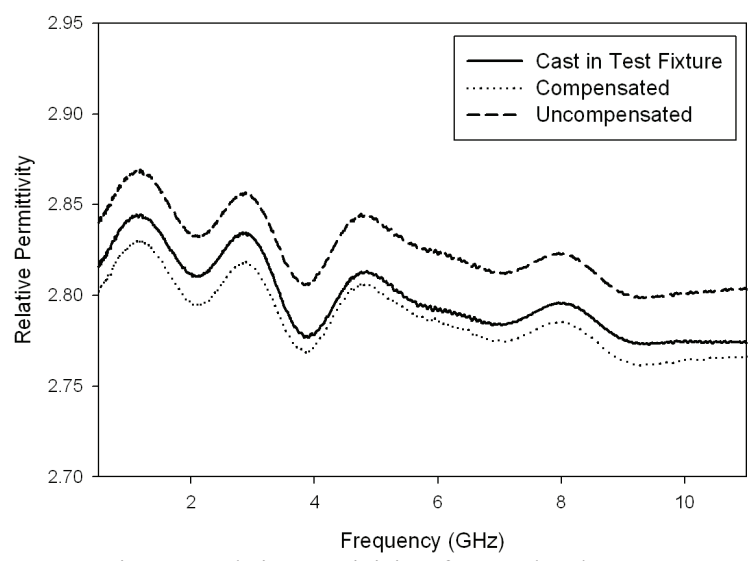

Figure 8. Relative permittivity of neat Sylgard ${ }^{\circledR} 184$

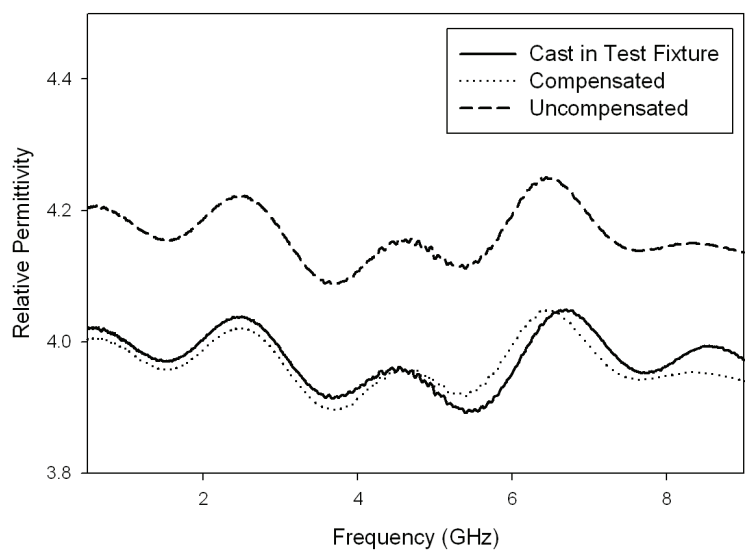

Figure 9. Relative permittivities of $10 \%$ volume loaded material.

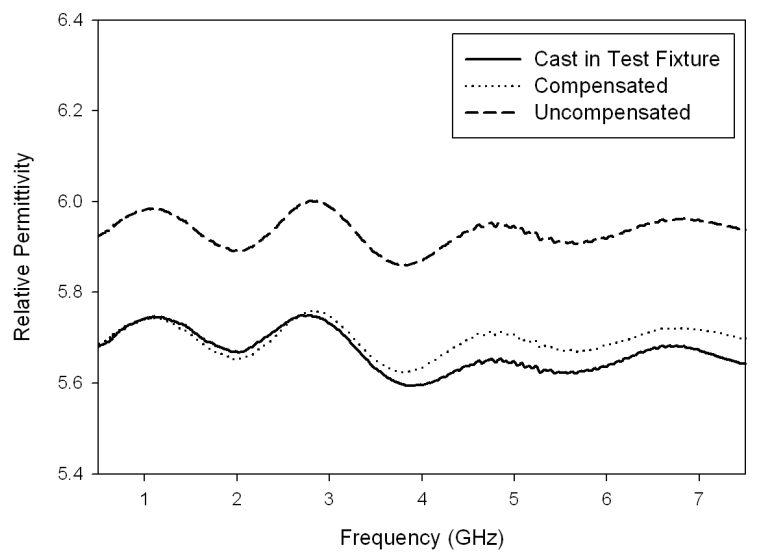

Figure 10. Relative permittivities of $20 \%$ volume loaded material.

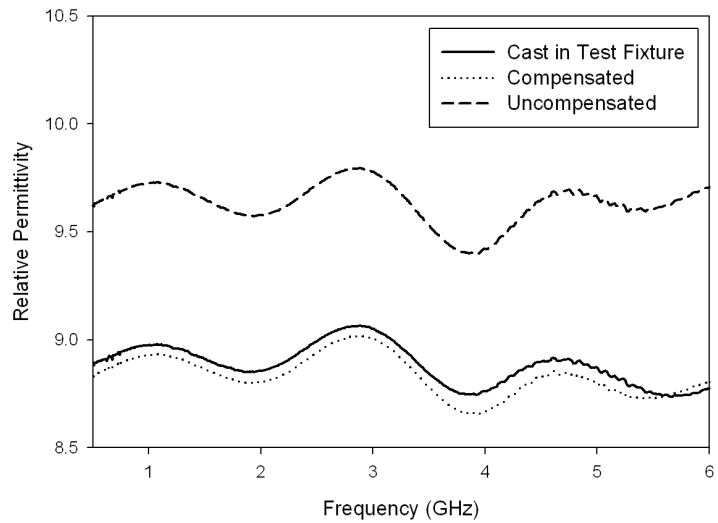

Figure 11. Relative permittivities of $30 \%$ volume loaded material.

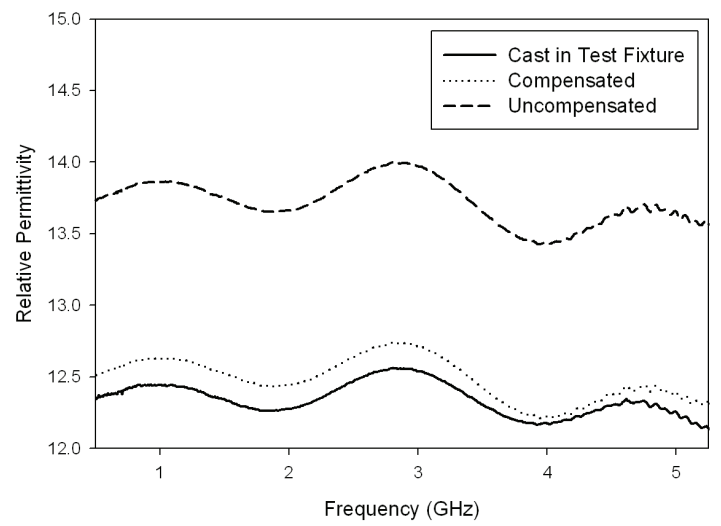

Figure 12. Relative permittivities of $40 \%$ volume loaded material

\section{CONCLUSION}

An approach to accurate permittivity measurements of compressible materials has been presented. The method uses both the measured, uncompressed density and the calculated, compressed density to account for the change in measured permittivity between compressed and uncompressed specimens of the same material. Normalizing susceptibility to compressed density provides data that closely agreed with actual values. Also, some of the advantages and difficulties in preparing elastomer and ceramic powder composite specimens have been discussed.

\section{REFERENCES}

[1] MU-EPSLN" for Windows Coaxial Line and Waveguide Material Measurements and Processing Software User's Guide Version 3.2, Damaskos, Inc., Concordville, PA, 2008.

[2] James Baker-Jarvis, "Transmission / Reflection and Short-circuit Line Permittivity Measurements," National Institute of Standards and Technology, Boulder, CO, NIST Technical Note 1341, July 1990.

[3] Information about Dow Corning ${ }^{\circledR}$ Brand Silicone Encapsulants, Dow Corning Corporation, Midland, MI, Form No. 10-898I-01, 2008.

[4] D. M. Pozar, Microwave Engineering, $3^{\text {rd }}$ ed. Hoboken, NJ: John Wiley \& Sons, Inc., 2005, pp. 9-10, 127-129.

[5] Matsuhaku. (2011, Jan 11). High precision density tester for solid \& liquid: $G P-120 S$ [Online]. Available:

http://www.matsuhaku.com/products/gp-120s.htm 